\title{
Modelling the Aggregated Dynamic Response of Electric Vehicles
}

\author{
Ziras, Charalampos; Hu, Junjie; You, Shi; Bindner, Henrik W.
}

Published in:

Proceedings of the 7th IEEE International Conference on Innovative Smart Grid Technologies

Link to article, DOI:

10.1109/ISGTEurope.2017.8260222

Publication date:

2017

Document Version

Peer reviewed version

Link back to DTU Orbit

Citation (APA):

Ziras, C., Hu, J., You, S., \& Bindner, H. W. (2017). Modelling the Aggregated Dynamic Response of Electric Vehicles. In Proceedings of the 7th IEEE International Conference on Innovative Smart Grid Technologies IEEE. https://doi.org/10.1109/ISGTEurope.2017.8260222

\section{General rights}

Copyright and moral rights for the publications made accessible in the public portal are retained by the authors and/or other copyright owners and it is a condition of accessing publications that users recognise and abide by the legal requirements associated with these rights.

- Users may download and print one copy of any publication from the public portal for the purpose of private study or research.

- You may not further distribute the material or use it for any profit-making activity or commercial gain

- You may freely distribute the URL identifying the publication in the public portal 


\title{
Modelling the Aggregated Dynamic Response of Electric Vehicles
}

\author{
Charalampos Ziras, Junjie Hu, Shi You, Henrik W. Bindner \\ Department of Electrical Engineering \\ Technical University of Denmark \\ Email: \{chazi, junhu, sy, hwbi\}@elektro.dtu.dk
}

\begin{abstract}
There is an increasing interest in the use of electric vehicles (EVs) for providing fast frequency reserves due to their large installed capacity and their very fast response. Most works focus on scheduling and optimization and usually neglect their aggregated dynamic response, which is particularly important from the power system perspective when EVs offer significant shares of such services. We present a literature review on the aggregated modelling of EVs and derive analytical expressions for the representation of $\mathrm{EV}$ populations based on the probability distributions of their parameters. Such approximations can be used in power system studies, in order to capture the dynamics of an EV population more accurately. Finally, we compare our approach to the most widely used in the literature, i.e. the averaging method where all EVs are represented with the population's average values, and discuss the key differences of the two approaches.
\end{abstract}

Index Terms-Aggregated dynamic response, electric vehicles aggregation, electric vehicles dynamics.

\section{INTRODUCTION}

The increasing penetration of renewable energy sources (RES) in the electricity generation mix and the electrification of heating and transportation challenge the traditional operation of the power system in a number of ways. First, they add more volatility in power generation; second, they decrease the system inertia due to the larger amounts of converter-connected loads and production units [1], [2]. The possibility of controlling loads for decreasing electricity costs and providing ancillary services to the system is a topic of extensive research. Thermostatically controlled loads [3], electric vehicles (EVs) [4], and battery energy storage systems (BESSs) [5] are the main types of units, also referred to as distributed energy resources (DERs), considered for such applications.

Despite the considerable amount of works focusing on optimization methods, scheduling and control of DERs for cost minimization and reserve provision, their dynamic behavior is still not so thoroughly investigated. In the majority of power system dynamics simulations, lumped models of populations of DERs are used to investigate their behavior and their impact on power system performance and stability [6], [7]. Indeed, the use of very large numbers of loads is inconvenient because the

C. Ziras, Y. Shi and H.W. Bindner would like to acknowledge the financial support of the EUDP project, Ecogrid 2.0, No 64015-0082. exact parameters of the DERs are usually unknown, whereas this increases the computational burden.

Even though the importance of aggregation in power system studies is acknowledged, it is found in the bibliography, that in most cases averaging methods are to express the DERs electrical dynamics. In this approach, average values of the parameters and the result is scaled up in order to characterize the aggregated response of the population; in some cases all of the individual units are modelled. A notable exception is the work of Moghadam et al. [8], [9], where the authors provide a closed-form representation of the power system dynamics after a disturbance, when DERs respond to frequency with randomized delays. In contrast to [8], [9], we focus on characterizing the aggregated response of a number of DERs to an input signal, when they present different response characteristics, in order to derive more accurate aggregated models. More specifically, the purpose of this paper is to investigate how accurate and under which conditions an averaging approach is, whereas we propose more accurate representations of populations of DERs based on limited population parameters knowledge.

The contribution of this paper is threefold. First, we present a literature review on the representation of the electrical dynamics of individual and aggregated portfolios of EVs. Second, we derive approximations in the time and Laplace domains of the most common transfer functions of EVs response, namely delays and first-order transfer functions, based on the statistical properties of their parameters. Third, we validate our approximations against the averaging method and the actual response of aggregations of EVs and we provide insight on the accuracy and behavior of the two approaches for various conditions.

The remainder of the paper is organized as follows. In Section II we review the dynamic representation of individual and aggregated EVs in a number of applications. In Section III we analytically derive approximations for the aggregated response of EVs in both the time and Laplace domains. In Section IV we present simulation results which benchmark the real response against the averaging and our proposed method, whereas Section V concludes.

\section{LITERATURE REVIEW}

In [10], a method to provide both primary frequency control (PFC) and inertial response with a population of EVs is 
proposed. In this work, the time delay introduced by the frequency measurement and each unit's response is considered; an average value of $150 \mathrm{~ms}$ for the delays is used and it is assumed that the delays are normally distributed between $100 \mathrm{~ms}$ and $200 \mathrm{~ms}$. However, a lumped model is not used and the EVs (which in this case are relatively few) are represented as individual loads. In [11] the performance of the interface, communication and control of an EV responding to local frequency measurements is assessed. In the conducted experiments, a $0.2 \mathrm{~s}$ delay due to frequency measurement was reported, along with some additional response delays. The total settling time was found to be $1 \mathrm{~s}$, implying first-order dynamics of the response, although not explicitly modelled.

The authors of [12] use an aggregated representation of each area's EV fleet in a four-area power system. In the first set of simulations, each fleet responds with a first-order transfer function (a gain and a time constant, which are not specified in the paper), whereas various communications delays are later introduced $(0.1 \mathrm{~s}, 0.2 \mathrm{~s}$ and $0.35 \mathrm{~s})$ to test the performance of the proposed distributed functional observers and finally the importance of those delays in system stability is highlighted. In [4] fleets of EVs are also modelled on an aggregated level with a first-order function in order to provide secondary frequency control; the gain is adjusted according to the state of charge and the time constant is set to $1 \mathrm{~s}$. The authors of [13] also represent an EV fleet with a first-order function and the time constants they calculate are based on the properties of the chargers and are equal to $35 \mathrm{~ms}, 50 \mathrm{~ms}$, and $100 \mathrm{~ms}$.

The authors of [14] study the effect of communication delays in the performance of EV aggregators in the PJM regulation market, where a mileage payment scheme is used. Both delays and first-order dynamics are used, where for analytical simplicity an average delay, an average gain and an average time constant are considered for all the EVs of each aggregator. The time constant is set to $100 \mathrm{~ms}$ and the authors show that the communication delay significantly affects the performance of frequency regulation offered by EV aggregators and can induce an unstable operation.

In [6] an aggregated EV model for providing PFC is also proposed. The authors model the dynamics with a first-order transfer function, but they use the average participation factor as a gain, which is calculated via the probability distribution function (PDF) of the EVs states of charge. In this case the gain is adaptive to the state of charge and in this way a representative gain for the population is used; the authors use an average value of $50 \mathrm{~ms}$ for the time constant and ignore the delays. In [15], series-produced EVs were tested to provide PFC in an islanded power system in a centralized manner, where frequency is not measured locally, but is routed via the internet. In the test, an overall delay of $2-3 \mathrm{~s}$ is observed for the newer EV models and the delay is even higher for the older models.

A state-space model to represent the system response of a homogeneous population of EV chargers under a hysteresisbased controller is proposed in [16]. In this method, the EVs state-of-charge is kept within a deadband, which is modulated so that a desired power trajectory is followed. The model captures the dynamic behavior of the population, but contrary to our work, the time scale used is much larger (therefore the model cannot be used to represent fast dynamics) and the population is homogeneous.

Finally, the authors of [17] analysed and tested small-scale EV batteries for direct load control. The settling time for a step change to the modelled battery system is equal to $70 \mathrm{~ms}$, implying a first-order time constant in the order of tens of ms. Uniformly distributed random delays from 0 to $1.1 \mathrm{~s}$ were used to account for communication delays, whereas the step response of 150 buildings equipped with BESSs was simulated. However, in the conducted case study only 2 BESSs were used, instead of a large population, and the values were scaled up.

As indicated from the literature review, it is widely accepted that the response of EVs can be modelled as a first-order transfer function with delay. In most cases the first-order time constant is relatively small, in the order of tens of ms, whereas there is a much larger diversity regarding the delays. This can be attributed to the wide range of applications and test setups, which can have a significant impact on the delays. Moreover, the dynamic representation of population of EVs is done via the averaging method, i.e. considering average values and then scaling up the result; in the following section we derive analytical expressions in order to more accurately represent the dynamics of a population of EVs.

\section{AgGREGATE DYNAMICS}

In this section we derive analytical expressions in the time and Laplace domains for the aggregated response of a population of loads which respond to the same input signal. More specifically, we present aggregated expressions for delayed responses, first-order responses and a more general case of a combination of both. In the rest of the paper we will use symbols $\mathbb{N}$ and $\mathbb{U}$ to denote the normal and uniform distribution respectively.

\section{A. Time delay}

Assume that the step response of a load $i$ is equal to $K_{\mathrm{g}, i} u\left(t-t_{\mathrm{d}, i}\right)$, with $t_{\mathrm{d}, i}$ and $K_{\mathrm{g}, i}$ representing the time delay and gain of each load respectively. In practice, the gain of a load's controller can be considered fixed, but the time delay will not necessarily be constant. We consider the case where the delay for each load can be well represented by its expected value and is therefore fixed as well.

If $t_{-}$is the minimum delay, $t_{+}$is the maximum delay, and $K_{\mathrm{tot}}=\sum_{i=1}^{N} K_{\mathrm{g}, i}$ is the sum of the individual gains of a population of $N$ loads, then the aggregate response in the time domain, $y(t)$, will be equal to

$$
y(t)= \begin{cases}0, & t<t_{-} \\ \sum_{i=1}^{N} K_{\mathrm{g}, i} u\left(t-t_{\mathrm{d}, i}\right), & t \leq t \leq t_{+} \\ K_{\mathrm{tot}}, & t>t_{+} .\end{cases}
$$


The summation in (1) at every point in time $t$ sums the gains for all loads with delays such that $t>t_{\mathrm{d}, i}$ (for a load with $t<$ $t_{\mathrm{d}, i}$ its contribution is equal to zero). This calculation requires full knowledge of the individual delays and gains. We want to approximate (1) with an expression that is more convenient and requires less information regarding the parameters of the population.

Let the time delays $t_{\mathrm{d}, i}$ be considered as independent and identically distributed (IID) random variables; the same assumption holds for the gains $K_{\mathrm{g}, i}$. We also assume that the delays and gains are independent, i.e. $t_{d, i} \perp K_{g, i}$, the variables are time-invariant, and the system is causal, i.e. the response at $t_{0}$ doesn't depend on the input for $t>t_{0}$.

We approximate (1) with its expected value. Note that products $K_{\mathrm{g}, i} u\left(t-t_{\mathrm{d}, i}\right)$ are also IID variables, as the products of $K_{\mathrm{g}, i}$, and $u\left(t-t_{\mathrm{d}, i}\right)$, which is also an IID variable as a monotonic function of $t_{\mathrm{d}, i}$; consequently, due to the independence of $t_{\mathrm{d}, i}$ and $K_{\mathrm{g}, i}$, it holds that $u\left(t-t_{\mathrm{d}, i}\right) \perp K_{\mathrm{g}, i}$. Since variables $K_{\mathrm{g}, i} u\left(t-t_{\mathrm{d}, i}\right)$ are IID, the expected value of the sum of $N$ such variables is equal to $N$ times the product of the expected value of each term [18]. For notation simplicity we drop $i$ and we refer to any IID variable without the subscript. The aggregate response can thus be approximated as

$$
y(t) \approx \begin{cases}0, & t<t_{-} \\ N \mathbb{E}\left[K_{\mathrm{g}}\right] \mathbb{E}\left[u\left(t-t_{\mathrm{d}}\right)\right], & t_{-} \leq t \leq t_{+} \\ N \mathbb{E}\left[K_{\mathrm{g}}\right], & t>t_{+}\end{cases}
$$

In (2) we replaced $K_{\text {tot }}$ with the expected value $\mathbb{E}\left[K_{\mathrm{g}}\right]$ of any of the IID variables $K_{\mathrm{g}, i}$, multiplied by $N$. Since $K_{\mathrm{g}, i}$ are time invariant, we also use the approximation $\sum_{i=1}^{N} K_{\mathrm{g}, i} \approx$ $N \mathbb{E}\left[K_{\mathrm{g}}\right]$. We define the PDF of the delays as $f_{t_{\mathrm{d}}}$ and the cumulative distribution function $(\mathrm{CDF})$ as $F_{t_{\mathrm{d}}}$. The expected value $\mathbb{E}\left[u\left(t-t_{\mathrm{d}}\right)\right]$ can be calculated as the expected value of a function of a random variable [18] for $t_{-} \leq t \leq t_{+}$as

$$
\mathbb{E}\left[u\left(t-t_{\mathrm{d}}\right)\right]=\int_{t_{-}}^{t_{+}} u\left(t-t_{\mathrm{d}}\right) f_{t_{\mathrm{d}}}(t) d t_{\mathrm{d}}
$$

Due to the properties of the step function inside the integral of (3), it holds that $\int_{t_{-}}^{t_{+}} u\left(t-t_{\mathrm{d}}\right) f_{t_{\mathrm{d}}}(t) d t_{\mathrm{d}}=\int_{t_{-}}^{t} f_{t_{\mathrm{d}}}(t) d t_{\mathrm{d}}=$ $F_{t_{\mathrm{d}}}(t)$. As a result, (2) can be written in a compact form as

$$
y(t) \approx N \mathbb{E}\left[K_{\mathrm{g}}\right] F_{t_{\mathrm{d}}}(t)
$$

Expression (4) is an approximation of the real response and is in general more convenient for two reasons. First, a Laplace transformation can be derived (if it exists), which is useful for power system dynamics studies. Second, an estimation of the real CDF can be used to express the aggregate dynamics, without full knowledge of the parameters. The aggregated transfer function $G(s)$ of the population is then approximated as $G(s)=N \mathbb{E}\left[K_{\mathrm{g}}\right] \mathscr{L}\left\{F_{t_{\mathrm{d}}}(t)\right\}$.
Next, we consider the case where $t_{\mathrm{d}} \sim \mathbb{U}\left[t_{\mathrm{a}}, t_{\mathrm{b}}\right]$, to derive analytical expressions for the aggregated response. The CDF of a uniform distribution is described by:

$$
F_{t_{d}}(t)= \begin{cases}0, & t<t_{\mathrm{a}} \\ \frac{t-t_{\mathrm{a}}}{t_{\mathrm{b}}-t_{\mathrm{a}}}, & t_{\mathrm{a}} \leq t \leq t_{\mathrm{b}} \\ 1, & t>t_{\mathrm{b}} .\end{cases}
$$

Using (5), equation (4) can be expressed in a compact form using the step funcion as

$$
y(t)=\frac{N \mathbb{E}\left[K_{\mathrm{g}}\right]}{t_{\mathrm{b}}-t_{\mathrm{a}}}\left[\left(t-t_{\mathrm{a}}\right) u\left(t-t_{\mathrm{a}}\right)-\left(t-t_{\mathrm{b}}\right) u\left(t-t_{\mathrm{b}}\right)\right] .
$$

Expression (6) shows that the system can be approximated by a one-input two-output system, whose outputs are summed, leading to a transfer function matrix of the form

$$
G(s)=\left[\begin{array}{c}
\frac{N \mathbb{E}\left[K_{\mathrm{g}}\right]}{t_{\mathrm{b}}-t_{\mathrm{a}}} \frac{1}{s} e^{-t_{\mathrm{a}} s} \\
-\frac{N \mathbb{E}\left[K_{\mathrm{g}}\right]}{t_{\mathrm{b}}-t_{\mathrm{a}}} \frac{1}{s} e^{-t_{\mathrm{b}} s}
\end{array}\right]
$$

\section{B. First-order response}

We now consider the case where the dynamics of each load can be expressed by a first order transfer function, with IID variables $t_{\mathrm{c}, i}$ representing the time constants of $N$ loads. For $t>0$, the step response of a single load $i$ is expressed by $K_{\mathrm{g}, i}\left(1-e^{-t / t_{\mathrm{c}, i}}\right)$. Following the same arguments and assumptions for parameters independence as in the case of the delays, we can approximate the aggregate response as follows

$$
y(t)=\sum_{i=1}^{N} K_{\mathrm{g}, i}\left(1-e^{-t / t_{\mathrm{c}, i}}\right) \approx N \mathbb{E}\left[K_{\mathrm{g}}\right]\left(1-\mathbb{E}\left[e^{-t / t_{\mathrm{c}}}\right]\right) .
$$

Let $t_{\mathrm{c}}$ follow a distribution with a PDF $f_{t_{\mathrm{c}}}$ and a $\operatorname{CDF} F_{t_{\mathrm{c}}}$; since $f_{t_{\mathrm{c}}}(t)=0$ for $t<0$, it holds that

$$
\mathbb{E}\left[e^{-t / t_{\mathrm{c}}}\right]=\int_{0}^{\infty} e^{-t / t_{\mathrm{c}}} f_{t_{\mathrm{c}}} d t_{\mathrm{c}}
$$

Therefore, the approximation of $y(t)$ can be written as

$$
y(t) \approx N \mathbb{E}\left[K_{\mathrm{g}}\right]\left(1-\int_{0}^{\infty} e^{-t / t_{\mathrm{c}}} f_{t_{\mathrm{c}}} d t_{\mathrm{c}}\right) .
$$

For most cases a closed form solution of (10) either does not exist, or the expression involves inconvenient terms, such as exponential integrals in the case of a uniform distribution, which result in logarithmic terms in the Laplace domain. However, due to the nature of the function $e^{-t / t_{\mathrm{c}}}$, the effect of $t_{\mathrm{c}}$ is relatively small and for a wide range of distributions, a very good approximation can be obtained by using the expected value of $t_{\mathrm{c}}, \mathbb{E}\left[t_{\mathrm{c}}\right]$. In the following we provide a proof for such an approximation in the Laplace domain.

Consider the first order transfer function $\frac{K_{\mathrm{g}, i}}{1+s t_{\mathrm{c}, i}}$ of load $i$ in the Laplace domain. If the step responses of the $N$ loads are aggregated, then the aggregate transfer function is equal to

$$
G(s)=\sum_{i=1}^{N} \frac{K_{\mathrm{g}, i}}{1+s t_{\mathrm{c}, i}} .
$$


As ratios of IID variables, the terms $\frac{K_{\mathrm{g}, i}}{1+s t_{\mathrm{c}, i}}$ are also IID variables and consequently $G(s)$ can be approximated by its expected value as

$$
G(s) \approx N \mathbb{E}\left[\frac{K_{g}}{1+s t_{\mathrm{c}}}\right] .
$$

As mentioned earlier, $\frac{K_{\mathrm{g}}}{1+s t_{\mathrm{c}}}$ is a random variable, and its distribution follows a ratio distribution. It can be approximated with a first order Taylor expansion [19] and by using the linearity property of the expectation $\mathbb{E}\left[1+s t_{\mathrm{c}}\right]=1+s \mathbb{E}\left[t_{\mathrm{c}}\right]$, we derive the following approximation

$$
G(s) \approx N \frac{\mathbb{E}\left[K_{\mathrm{g}}\right]}{1+s \mathbb{E}\left[t_{\mathrm{c}}\right]}
$$

\section{Combined response of first order and delays}

In many applications, the response of the load is subject to a combination of delays and first-order transfer functions. In some cases more complicated transfer functions can describe the dynamics of the loads, but in the majority of power system applications time delays and first-order functions are used. A typical example is the response of an electric vehicle or a battery energy storage system subject to a set-point change.

In the previous subsections we presented approximations for the summation of first-order transfer functions and delays. Typically, a number of delays which represent various processes, i.e. frequency measurement, communication delay and controller delay, and a first-order function, which represents the dynamic behavior of the load, are used to describe the loads response.

Consider a load $i$ whose response in the Laplace domain is described as the product of a delay $e^{-t_{\mathrm{a}, i} s}$, an additional delay $e^{-t_{\mathrm{b}, i} s}$ and a first-order transfer function $\frac{K_{g, i}}{1+s t_{\mathrm{c}, i}}$. Based on our approach, due to the assumption of parameters independence, the aggregated transfer function for a population of $N$ loads will be the product of $N \frac{\mathbb{E}\left[K_{\mathrm{g}}\right]}{1+s \mathbb{E}\left[t_{\mathrm{c}}\right]}$ and the Laplace transformations $\mathscr{L}\left\{F_{t_{\mathrm{a}}}(t)\right\}$ and $\mathscr{L}\left\{F_{t_{\mathrm{b}}}(t)\right\}$, where $F_{t_{\mathrm{a}}}$ and $F_{t_{\mathrm{b}}}$ represent the CDFs of $t_{\mathrm{a}}$ and $t_{\mathrm{b}}$ respectively.

At this point, it is important to note that the first-order terms are expressed via the estimated mean value of the parameters, as in the averaging method, and only the delay terms are different, due to the use of the CDFs in the approximations, instead of the estimated mean values. In the following section we provide simulation results for a number of common distributions to validate the accuracy of our approximations and benchmark them against the averaging method.

\section{Simulation RESUlts}

In this section we present simulation results to validate the accuracy of the derived approximations and show how the two aggregation approaches (the averaging method and the proposed) represent the dynamics of a population of electric vehicles. We first examine the summation of first-order responses $\frac{K_{\mathrm{g}, i}}{1+s t_{\mathrm{c}, i}}$. In [13] it was stated that the time constants of the first-order transfer functions of electric vehicles are in the range of $35-100 \mathrm{~ms}$. In the case of an $\mathrm{EV}$, the gain represents the charger's gain, which allows the EV charger to modify its power output linearly with the input signal; we treat the input signal as a normalized signal $\in[-1,1]$ and in this case the gain would be expressed in $\mathrm{kW}$. The results for 100 loads are shown in Fig. 1, where uniform and normal distributions are considered for the parameters.
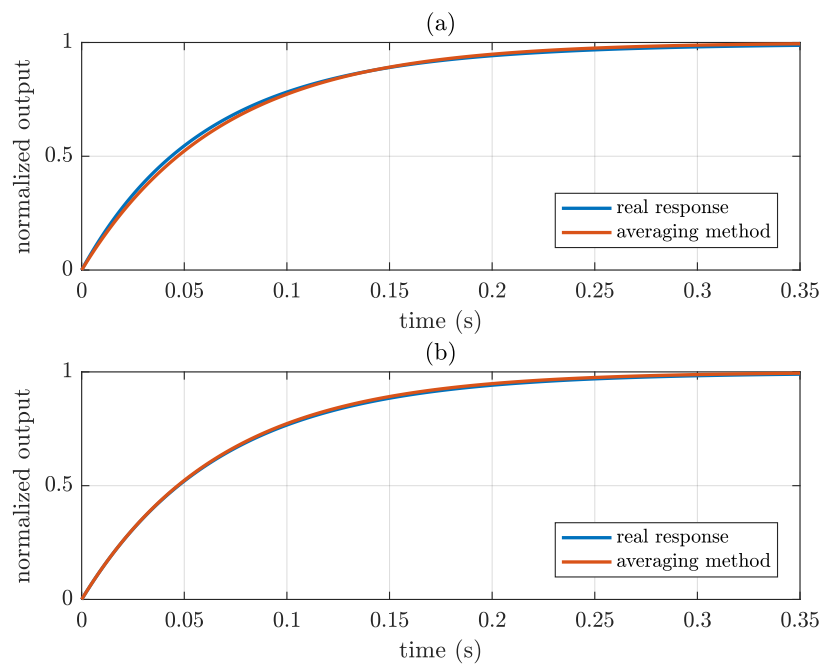

Fig. 1: Real and aggregated step response of 100 loads for two cases: (a) $K_{\mathrm{g}} \sim \mathbb{U}[8,12] \mathrm{kW}, t_{\mathrm{c}} \sim \mathbb{U}[0.035,0.1] \mathrm{s}$, and (b) $K_{\mathrm{g}} \sim \mathbb{N}[10,0.8] \mathrm{kW}, t_{\mathrm{c}} \sim \mathbb{N}[0.0675,0.01] \mathrm{s}$.

From the step responses presented in Fig. 1, it can be seen that the averaging method works very well for both uniform and normal distributions for the first-order constants. This is in accordance with our results, as expressed via equation (13). We further conducted extensive simulations for a wide range of distributions and parameters and we verified that the averaging method provides a very accurate approximation.

Next, we examined a more common representation of the dynamic behavior of EVs, namely a delayed first-order response of the form $e^{-t_{\mathrm{d}, i} s} \frac{K_{\mathrm{g}, i}}{1+s t_{\mathrm{c}, i}}$. Since the averaging method gives very accurate results for first-order responses, we fixed the distributions of $K_{\mathrm{g}}$ and $t_{\mathrm{c}}$ to the normal distributions used in the previous example and we examined the effect of the time delays.

Figure 2 shows the real response and the two approximations in the case of uniformly distributed delays. From our simulations we observed that the range of the distribution significantly affects the accuracy of the averaging method. In the case of narrowly distributed parameters, the average value is representative of the delays, and we obtain relatively accurate approximations. As the range of the distribution increases, the response of the averaging method deviates more from the real response.

Most importantly, instead of gradually increasing, the output increases much more steeply around the average delay value. In case of dynamic frequency simulations, such a response would most probably result in different dynamic performance and lead us to false conclusions. Additionally, due to the presence of the first-order response, the averaging method is 

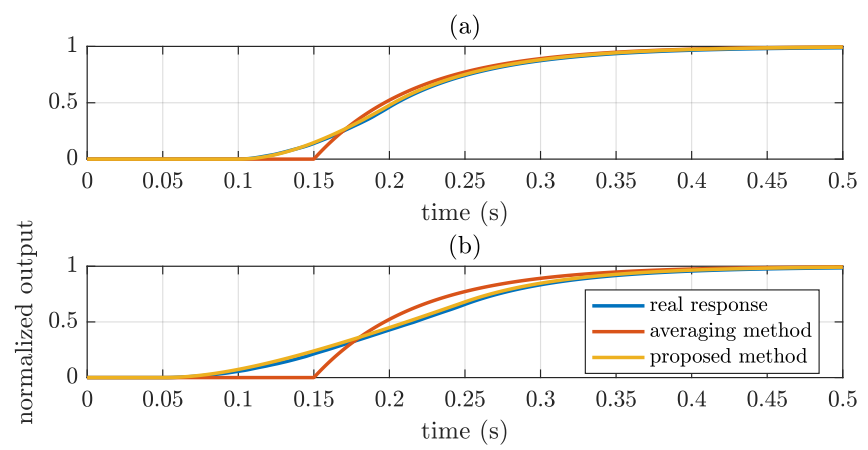

(c)

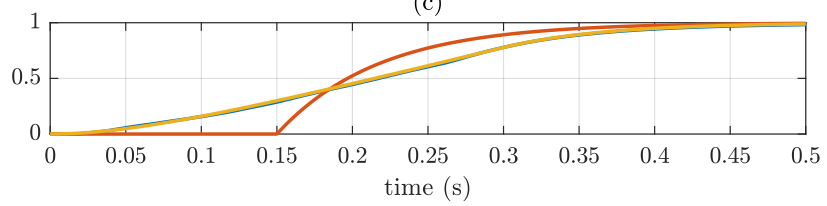

Fig. 2: Real and aggregated step response of 100 loads for three cases: (a) $t_{\mathrm{d}} \sim \mathbb{U}[0.1,0.2] \mathrm{s}$, (b) $t_{\mathrm{d}} \sim \mathbb{U}[0.05,0.25] \mathrm{s}$, and (c) $t_{\mathrm{d}} \sim \mathbb{U}[0,0.3] \mathrm{s}$.

able to result in a better accuracy for the time period closer to the settling value. If only the delays were present, the real response would be very close to a ramping response, and in that case the averaging method would result in a step response, an approximation which would be considerably different from the actual one. As can be seen in Fig. 2, in all cases where the delays are uniformly distributed, our proposed approximation is very close to the actual response of the loads.

In Fig. 3 we present the results for the case where the time delays are normally distributed. We must note that we have not presented an analytical expression for the aggregate transfer function in this case, since there is no closed form expression for the Laplace transformation of the Gaussian distribution. However, the aggregate transfer function matrix (7) was used, where $t_{\mathrm{b}}$ was set equal to the mean value of $t_{\mathrm{d}}$ plus two times the standard deviation, and $t_{\mathrm{a}}$ was set equal to the mean value minus two times the standard deviation.

Again we observe the beneficial impact of the first-order response, which improves the approximation of the averaging method. For narrow normal distributions the averaging method provides a good approximation, but as the standard deviation increases, this method results in significantly inaccurate responses. On the other hand, the simple approximation we proposed is able to capture the dynamics of the population in a much more accurate manner, as seen in Fig. 3.

In the case of delays, the response obtained with the averaging method can result in significantly different dynamic behaviors, because the aggregation will exhibit a larger delay, combined with a faster response. In reality, due to the delays distribution, the aggregation will respond more smoothly and with a smaller delay. For this reason we believe that an accurate representation of the dynamics is required, especially when conducting power system stability studies, since it has been shown that relatively small delays combined with fast
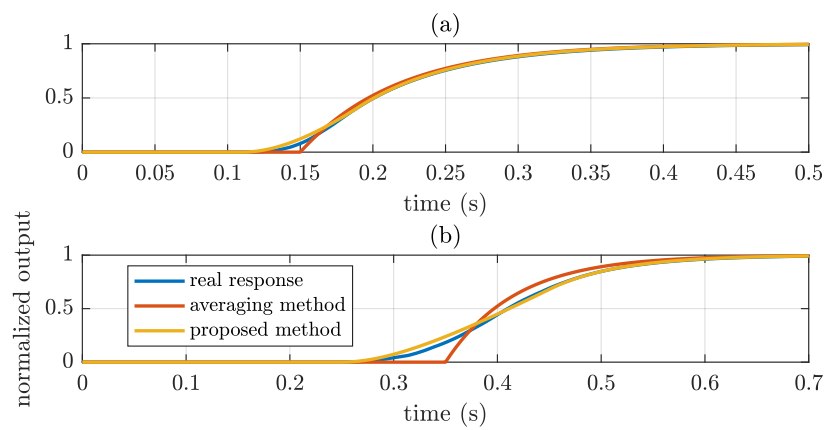

(c)

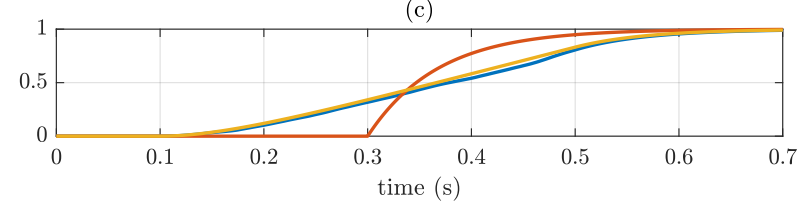

Fig. 3: Real and aggregated step response of 100 loads for three cases: (a) $t_{\mathrm{d}} \sim \mathbb{N}[0.15,0.02] s$, (b) $t_{\mathrm{d}} \sim \mathbb{N}[0.35,0.05] s$, and (c) $t_{\mathrm{d}} \sim \mathbb{N}[0.5,0.1] \mathrm{s}$.

responses from loads offering PFC can lead to instability [20].

In order to provide a more comprehensive understanding of how the distribution of the delays affects the two approximation methods, we conducted a sensitivity analysis. More specifically, we considered a uniform distribution for the delays with varying mean values and ranges and normal distributions for the first-order transfer function parameters, as in the previous 2 examples. The range is expressed with a normalized number against the mean value, i.e. a range of $50 \%$ with a mean delay of $0.5 \mathrm{~s}$ corresponds to a uniform distribution with a minimum value of $0.25 \mathrm{~s}$ and a maximum value equal to $0.75 \mathrm{~s}$. We used the Euclidean norm to express the errors of the approximations compared to the real response and the results are presented in Fig. 4 and Fig. 5.

The errors are expected to increase for larger values of the delays in absolute terms. Fig. 4 shows the strong correlation of the error with the range of the distribution when the averaging method is used. Even for large average delays, the errors are relatively small for narrow distributions, because in that case the average value is representative of the population. As the range increases, particularly for larger average values, the averaging method performs very poorly and the approximation gives very inaccurate results. On the other hand, the proposed method produces much smaller errors (up to one order of magnitude) for all the examined cases, which are mainly attributed to the population size (the simulated distributions are not perfectly uniform distributions), as seen in Fig. 5.

\section{CONClusion}

We have conducted a literature review on the dynamic representation of EVs on an individual and aggregated level, and we showed that a delayed first-order response is the widely accepted model for the dynamic behavior. We presented a methodology to derive analytical expressions in the time and Laplace domains for aggregations of EVs and we showed 


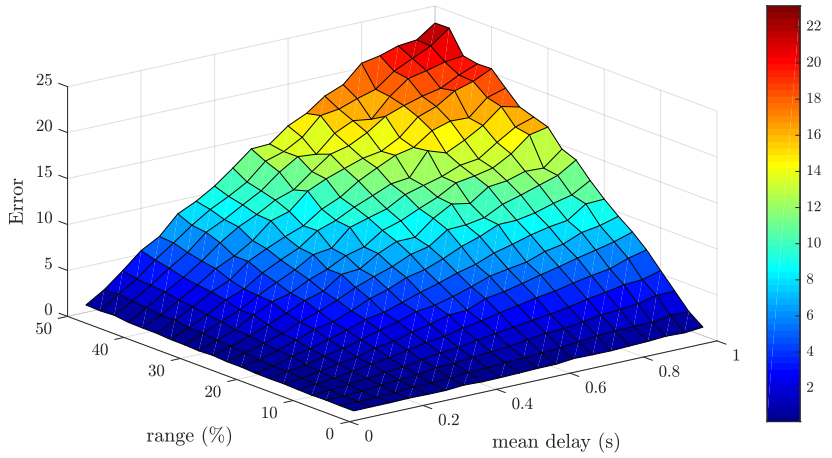

Fig. 4: Approximation errors of the averaging method for different parameters of the uniformly distributed time delay.

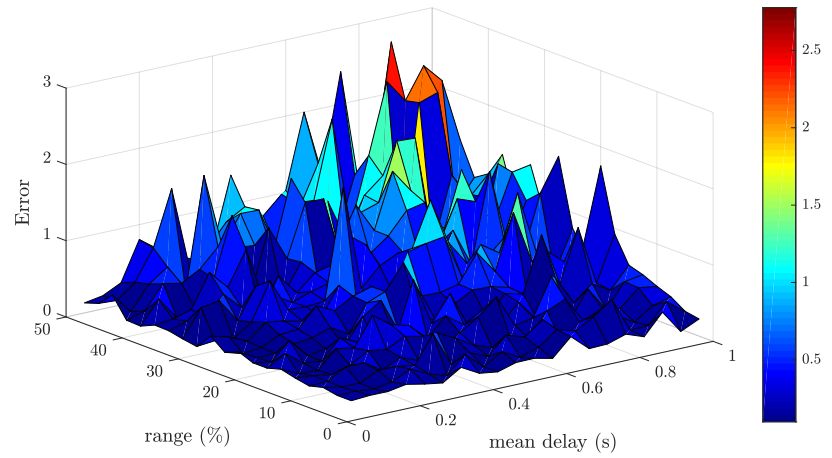

Fig. 5: Approximation errors of the proposed method for different parameters of the uniformly distributed time delay.

that a very good accuracy can be achieved by using the population's average values in the case of a first-order function. However, using the average value in the case of time delays results in significant errors, especially as the distribution of the delays becomes wider. More specifically, the averaging method results in a much steeper response, compared to the actual. For instance, if the delays are uniformly distributed, the aggregated response (and our proposed approximation) is a ramp-response, whereas the averaging method results in a step response at the average delay value. In that case, our proposed approximation can drastically reduce the errors and provide a much more accurate representation of the dynamics. It is also interesting to note that the presence of a first-order transfer function improves the approximation of the averaging method in the case of delays, because it results in a response which to an extent resembles the ramping response close to the settling time, as seen in Fig. 3. For purely delayed responses, or with very small first-order time constants, the averaging method results in a significantly different response, compared to the actual.

The proposed methodology can be used to derive very accurate transfer functions for populations of EVs, with limited knowledge about their parameters. These transfer functions can be used in power system dynamics studies or for evaluating the tracking of reference signals (for instance in load frequency control) by populations of EVs.

\section{REFERENCES}

[1] G. Pepermans, J. Driesen, D. Haeseldonckx, R. Belmans, W. Dhaeseleer, Distributed generation: definition, benefits and issues, Energy Policy, vol. 33, Issue 6, pp. 787-798, April 2005

[2] P. De Martini, K.M. Chandy, N.A. Fromer (2012) Grid 2020: Towards a policy of renewable and distributed energy resources. Resnick Institute, California Institute of Technology, Pasadena

[3] H. Hao, Y. Lin, A. S. Kowli, P. Barooah and S. Meyn, ”Ancillary Service to the Grid Through Control of Fans in Commercial Building HVAC Systems," in IEEE Transactions on Smart Grid, vol. 5, no. 4, pp. 20662074, July 2014

[4] S. Vachirasricirikul and I. Ngamroo, "Robust LFC in a Smart Grid With Wind Power Penetration by Coordinated V2G Control and Frequency Controller," in IEEE Transactions on Smart Grid, vol. 5, no. 1, pp. 371380, Jan. 2014

[5] G. He, Q. Chen, C. Kang, P. Pinson and Q. Xia, ”Optimal Bidding Strategy of Battery Storage in Power Markets Considering PerformanceBased Regulation and Battery Cycle Life," in IEEE Transactions on Smart Grid, vol. 7, no. 5, pp. 2359-2367, Sept. 2016

[6] S. Izadkhast, P. Garcia-Gonzalez and P. Fras, "An Aggregate Model of Plug-In Electric Vehicles for Primary Frequency Control," in IEEE Transactions on Power Systems, vol. 30, no. 3, pp. 1475-1482, May 2015

[7] J. R. Pillai and B. Bak-Jensen, "Integration of Vehicle-to-Grid in the Western Danish Power System,' in IEEE Transactions on Sustainable Energy, vol. 2, no. 1, pp. 12-19, Jan. 2011

[8] M. R. Vedady Moghadam, R. T. B. Ma and R. Zhang, "Distributed Frequency Control in Smart Grids via Randomized Demand Response," in IEEE Transactions on Smart Grid, vol. 5, no. 6, pp. 2798-2809, Nov. 2014

[9] M. R. V. Moghadam, R. Zhang and R. T. B. Ma, 'Distributed Frequency Control via Randomized Response of Electric Vehicles in Power Grid,' in IEEE Transactions on Sustainable Energy, vol. 7, no. 1, pp. 312-324, Jan. 2016

[10] P.M. Rocha Almeida, F.J. Soares, J.A. Peas Lopes, Electric vehicles contribution for frequency control with inertial emulation, Electric Power Systems Research, vol. 127, pp. 141-150, Oct. 2015

[11] Yutaka Ota, Haruhito Taniguchi, Jumpei Baba, Akihiko Yokoyama, Implementation of autonomous distributed V2G to electric vehicle and DC charging system, Electric Power Systems Research, vol. 120, pp. 177-183, Mar. 2015

[12] T. N. Pham, H. Trinh and L. V. Hien, "Load Frequency Control of Power Systems With Electric Vehicles and Diverse Transmission Links Using Distributed Functional Observers," in IEEE Transactions on Smart Grid, vol. 7, no. 1, pp. 238-252, Jan. 2016

[13] Y. Mu, J. Wu, J. Ekanayake, N. Jenkins and H. Jia, "Primary Frequency Response From Electric Vehicles in the Great Britain Power System," in IEEE Transactions on Smart Grid, vol. 4, no. 2, pp. 1142-1150, June 2013

[14] K. Ko; S. Han; D. K. Sung, "A New Mileage Payment for EV Aggregators with Varying Delays in Frequency Regulation Service," in IEEE Transactions on Smart Grid, vol.PP, no.99, pp.1-1

[15] M. Marinelli, S. Martinenas, K. Knezović, P. B. Andersen, "Validating a centralized approach to primary frequency control with series-produced electric vehicles", Journal of Energy Storage, vol 7, pp. 63-73, Aug. 2016

[16] S. Kundu and I. A. Hiskens, "State-space modelling of hysteresis-based control schemes," 2013 European Control Conference (ECC), Zurich, 2013, pp. 2535-2540

[17] Y. J. Kim, G. Del-Rosario-Calaf and L. K. Norford, "Analysis and Experimental Implementation of Grid Frequency Regulation Using Behindthe-Meter Batteries Compensating for Fast Load Demand Variations,' in IEEE Transactions on Power Systems, vol. 32, no. 1, pp. 484-498, Jan. 2017

[18] D. J. Olive, Statistical Theory and Inference, Springer International Publishing, 2014

[19] G. Van Kempen and L. Van Vliet, Mean and variance of ratio estimators used in fluorescence ratio imaging, Cytometry, vol. 39, no. 4, pp. 300 305,2000

[20] C. Ziras, E. Vrettos, and S. You, Controllability and stability of primary frequency control from thermostatic loads with delays, J. Mod. Power Syst. Clean Energy, vol. 5, no. 1, pp. 43-54, 2017 